\title{
Magnetic Properties and Core Loss Behavior of Fe-6.5wt.\%Si Ribbons Prepared by Melt Spinning
}

\author{
S. Wang, Y. M. Jiang, Y. F. Liang, F. Ye, and J. P. Lin \\ State Key Laboratory for Advanced Metals and Materials, University of Science and Technology Beijing, Beijing 100083, China \\ Correspondence should be addressed to Y. F. Liang; liangyf@skl.ustb.edu.cn
}

Received 13 November 2014; Revised 31 December 2014; Accepted 2 January 2015

Academic Editor: Pavel Lejcek

Copyright ( 2015 S. Wang et al. This is an open access article distributed under the Creative Commons Attribution License, which permits unrestricted use, distribution, and reproduction in any medium, provided the original work is properly cited.

Fe-6.5wt.\%Si alloy is prepared in the form of continuous ribbons with $25 \mathrm{~mm}$ in width and $0.03 \mathrm{~mm}$ in thickness by using melt spinning technique. The ribbons are flexible and could be wounded into tapes. DC magnetic properties and core loss behaviors of the ribbons after heat treatment are investigated in this paper. The magnetic properties are compared with ribbons by cold rolling and CVD methods. The melt spinning ribbons exhibit much less core loss in the frequencies more than $10 \mathrm{kHz}$. The melt spinning ribbons are promising to be used for electric devices used in medium or higher frequencies.

\section{Introduction}

High silicon steels (Si content $\geq 4.5 \mathrm{wt} . \%$ ) have been considered as potential materials for application in distribution transformers owing to their excellent soft magnetic properties [1]. Particularly high silicon steel containing 6.5wt.\%Si has potential applications in magnetic devices due to its high permeability, high magnetization, high Curie temperature, and very low magnetostriction, as well as low core loss. However, it has not been used widely because of its poor mechanical workability at room temperature. The fabrication of Fe-6.5wt.\%Si ribbons through conventional rolling process is difficult owing to inherent brittleness of the material [2]. The brittleness is due to the formation of ordered phases and coarse grains $[3,4]$. It can be overcome by the rapid solidification technique like melt spinning. Enokizono et al. successfully prepared Fe-6.5wt.\%Si ribbons with 0.01$0.04 \mathrm{~mm}$ in thickness and about $1-2 \mathrm{~mm}$ in width [5].

In the present work, we obtained ribbons with $0.03 \mathrm{~mm}$ in thickness and $25 \mathrm{~mm}$ in width prepared by melt spinning technique. Magnetic properties and core loss behaviors are studied in this paper.

\section{Experiments}

Fe-6.5wt.\%Si master alloy was prepared by melting iron and metallic silicon ( $99.5 \%$ purity) in a vacuum induction furnace and then poured into an ingot mold. Table 1 shows the chemical analysis of the alloy.

The Fe-6.5wt.\%Si ribbons were prepared by melt spinning technique. A melt spinning equipment mainly includes medium-frequency induction furnace, nozzle, and copper roller cooled by water. In the process of preparing ribbons, the alloy of Fe-6.5wt.\%Si was put in the medium-frequency induction furnace and was melted. A stream of the molten alloy was ejected onto the surface of the copper roller through the nozzle by adding a constant pressure in the furnace. The molten alloy was then quenched rapidly by the copper roller and formed into a long ribbon.

By optimizing the size of the nozzle, the ejection pressure, the temperature of the molten alloy, the space between nozzle and copper roller, and the speed of rotation roller, the ribbons were successfully prepared in the form of $20 \mathrm{~mm}$ in width and $0.03 \mathrm{~mm}$ in thickness and could be wounded into tapes, as showed in Figure 1. Surface quality of the ribbon is good and the two edges of the ribbon are straight. These ribbons are flexible and could be bent into $180^{\circ}$ without crack.

For measurement of the magnetic properties, the ribbons were cut into $10 \mathrm{~mm}$ wide, coated by $\mathrm{MgO}$ powders, and wound into toroidal core. The inner and the outer diameter of the core were $15 \mathrm{~mm}$ and $23 \mathrm{~mm}$, respectively, as shown in Figure 2. 


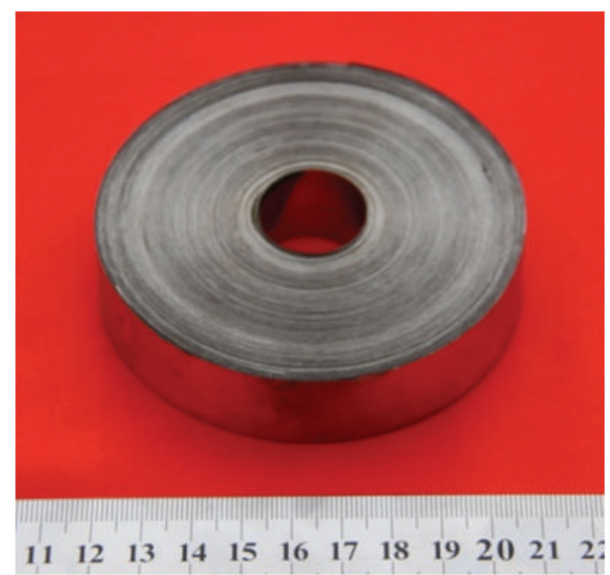

FIGURE 1: Fe-6.5wt.\%Si ribbons with $25 \mathrm{~mm}$ in width and $0.03 \mathrm{~mm}$ in thickness prepared by melt spinning.

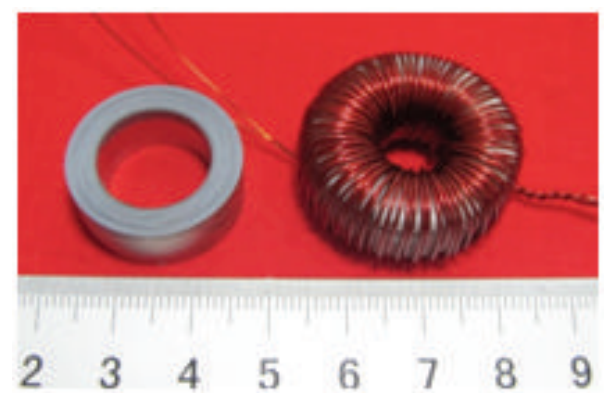

Figure 2: Wounded toroidal cores by Fe-6.5wt.\%Si ribbons, before (left) and after (right) wounding copper wires for magnetic testing.

TABLE 1: Chemical composition of Fe-6.5wt.\%Si alloy.

\begin{tabular}{lccccccc}
\hline Element & $\mathrm{Si}$ & $\mathrm{C}$ & $\mathrm{Mn}$ & $\mathrm{P}$ & $\mathrm{S}$ & $\mathrm{B}$ & $\mathrm{Fe}$ \\
\hline wt.\% & 6.46 & 0.0034 & 0.04 & 0.0056 & 0.0013 & 0.0005 & The rest \\
\hline
\end{tabular}

It is reported that the soft magnetic properties of the ribbons are degraded by stresses produced during the production process [6], while $\mathrm{B} 2$ and $\mathrm{D}_{3}$ order phases are suppressed [7]. The ordered phases are helpful to improve magnetic properties [8]. So the core should be annealed for improving magnetic properties. In this work, the toroidal core was annealed at $1100^{\circ} \mathrm{C}$ for 5 hours in vacuum and then cooled in furnace to room temperature. For measurement, the annealed cores were put in nylon case to avoid further stress and then primary and secondary copper wires were wound, as shown in Figure 2.

The structure of the ribbons before and after heat treatment was studied using X-ray diffractometry (XRD, Rigaku, Ultima IV) and transmission electron microscopy (TEM, FEI Tecnai F30). The AC and DC magnetic properties of the cores were measured using NIM-2000S AC instrument and NIM3000S DC instrument, respectively. The AC and DC magnetic

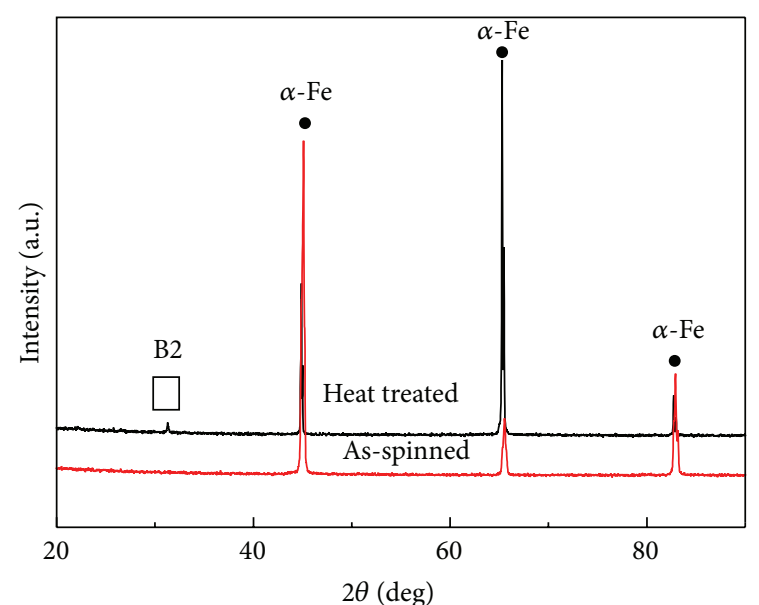

FIGURE 3: XRD patterns of melt spinned specimens before and after heat treatment $\left(1100^{\circ} \mathrm{C} / 5 \mathrm{~h}+\mathrm{FC}\right)$.

properties are compared with the magnetic properties of ribbons produced by cold rolling and CVD methods.

\section{Results and Discussion}

3.1. Microstructural Characteristics. Figure 3 presents the XRD results of melt spinned specimens before and after heat treatment. B2 reflection is not observed in as-spinned specimens, while a weak B2 refection can be observed after heat treatment.

The phase evolution can be also confirmed from the TEM diffraction patterns in Figures 4 and 5 with bright-field TEM micrograph and the corresponding SADP. It is found that there is no B2 phase in as-spinned specimens as shown in Figure 4, while the appearance of B2 phase is obvious after heat treatment as shown in Figure 5.

3.2. DC Magnetic Properties. DC magnetic properties of the ribbons after heat treatment were measured in static magnetic field, as shown in Table 2 compared with the ribbons prepared by cold rolling and CVD methods. $B_{8}, B_{25}$, and $B_{50}$ are maximum magnetic flux density measured at the DC magnetic field of $800 \mathrm{~A} / \mathrm{m}, 2500 \mathrm{~A} / \mathrm{m}$, and $5000 \mathrm{~A} / \mathrm{m}$, respectively.

The values of $B_{8}$ and $B_{25}$ are very close between melt spinned ribbons and CVD ribbons. The relative permeability $\left(\mu_{r}\right)$ of the melt spinned ribbon is only about half of CVD ribbon. The reason is mainly due to the ultrathin thickness of the melt spinned ribbons. Coercivity of the melt spinned ribbon is as good as cold rolled one. The DC hysteresis loop was measured at a magnetic field up to $H=10000 \mathrm{~A} / \mathrm{m}$, which is shown in Figure 6.

3.3. AC Magnetic Properties. Table 3 shows the core losses of melt spinned ribbons compared with ribbons from cold rolling and CVD methods. It is found that melt spinned ribbons exhibit less core losses than cold rolled and CVD ones 


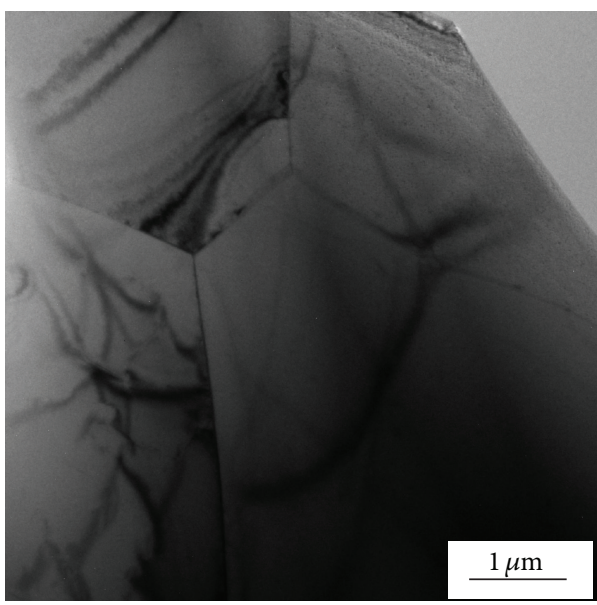

(a)

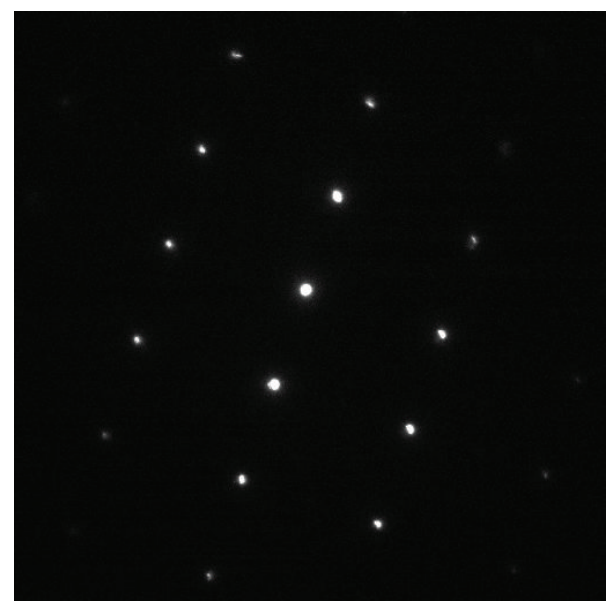

(b)

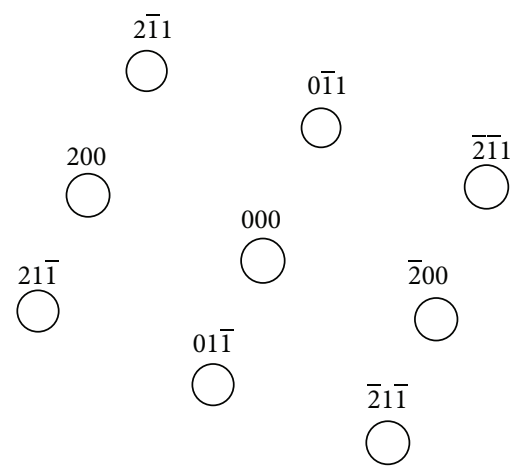

(c)

FIGURE 4: TEM micrographs of the as-spinned Fe-6.5wt.\%Si ribbons. (a) Bright-field image; (b) SADP of the zone axis of [001]; (c) the corresponding schematic illustration, where only A2 phase is identified.

TABLE 2: DC magnetic properties of ribbons produced by melt spinning.

\begin{tabular}{|c|c|c|c|c|c|c|c|}
\hline & \multirow{2}{*}{ Thickness (mm) } & \multicolumn{3}{|c|}{ Magnetic flux density (T) } & \multirow{2}{*}{$\begin{array}{c}\text { Residual magnetic } \\
\text { flux } \\
\text { density }(\mathrm{T}) \\
B_{r}\end{array}$} & \multirow{2}{*}{$\begin{array}{c}\text { Relative } \\
\text { permeability } \\
\mu_{r}\end{array}$} & \multirow{2}{*}{$\begin{array}{c}\text { Coercivity } \\
(\mathrm{A} / \mathrm{m}) \\
H_{c}\end{array}$} \\
\hline & & $B_{8}$ & $B_{25}$ & $B_{50}$ & & & \\
\hline $\begin{array}{l}\text { Melt spinning } \\
\text { ribbon }\end{array}$ & 0.03 & 1.27 & 1.38 & 1.50 & 1.12 & 11234 & 35.65 \\
\hline $\begin{array}{l}\text { Cold rolling } \\
\text { ribbon [9] }\end{array}$ & 0.05 & 1.40 & - & - & - & 11200 & 40.83 \\
\hline $\begin{array}{l}\text { CVD } \\
\text { ribbon [4] }\end{array}$ & 0.1 & 1.29 & 1.40 & - & - & 23000 & - \\
\hline
\end{tabular}

TABLE 3: Core losses of melt spinned ribbons compared with ribbons from cold rolling and CVD.

\begin{tabular}{llcccccccccc}
\hline Method & $\begin{array}{l}\text { Thickness } \\
(\mathrm{mm})\end{array}$ & $P_{1 / 400}$ & $P_{0.5 / 1 \mathrm{~K}}$ & $P_{0.1 / 5 \mathrm{~K}}$ & $P_{0.2 / 5 \mathrm{~K}}$ & $P_{0.05 / 10 \mathrm{~K}}$ & $P_{0.1 / 10 \mathrm{~K}}$ & $P_{0.05 / 20 \mathrm{~K}}$ & $P_{0.07 / 20 \mathrm{~K}}$ & $P_{0.07 / 30 \mathrm{~K}}$ & $P_{0.07 / 40 \mathrm{~K}}$ \\
\hline Melt spinned & 0.03 & 7.31 & 5.47 & 1.90 & 6.99 & 1.21 & 4.62 & 3.13 & 6.03 & 10.86 & 16.69 \\
Cold rolled & 0.05 & 8.66 & 6.3 & - & 8.82 & - & 6.23 & - & 8.81 & 16.5 & 26.1 \\
CVD & 0.05 & 6.1 & 4.6 & - & 6.2 & - & 5.1 & - & 7.0 & 12.7 & 20.5 \\
\hline
\end{tabular}




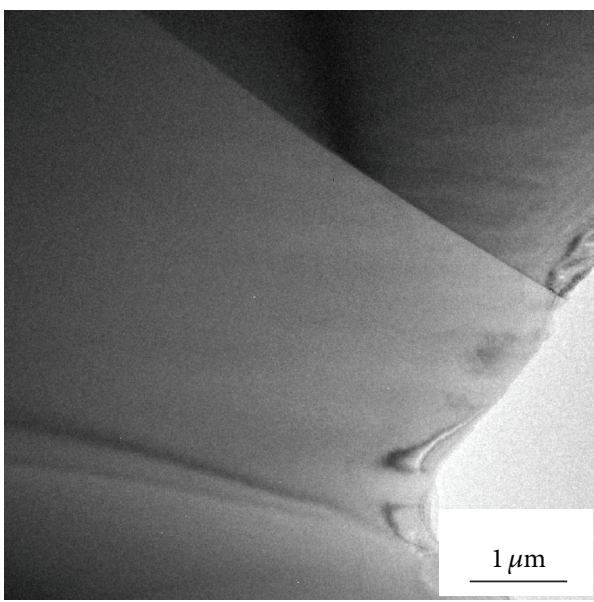

(a)

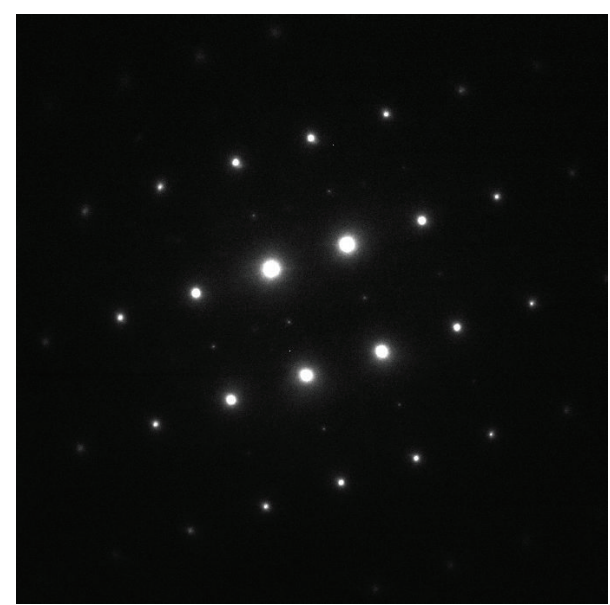

(b)

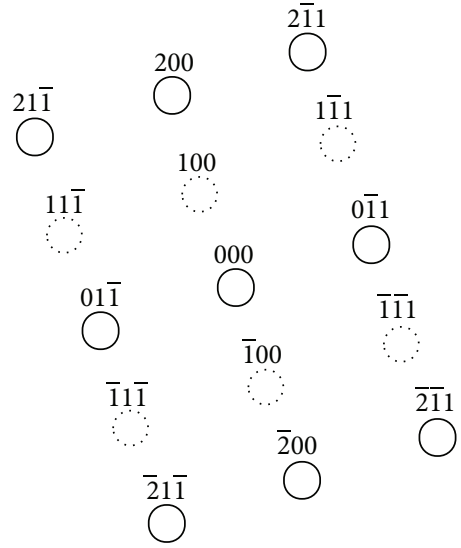

(c)

FIGURE 5: TEM micrographs of the heat treated Fe-6.5wt.\%Si ribbons. (a) Bright-field image; (b) SADP of the zone axis of [001]; (c) the corresponding schematic illustration, where solid circles represent A2 phase, and the dotted circles represent B2 phase.

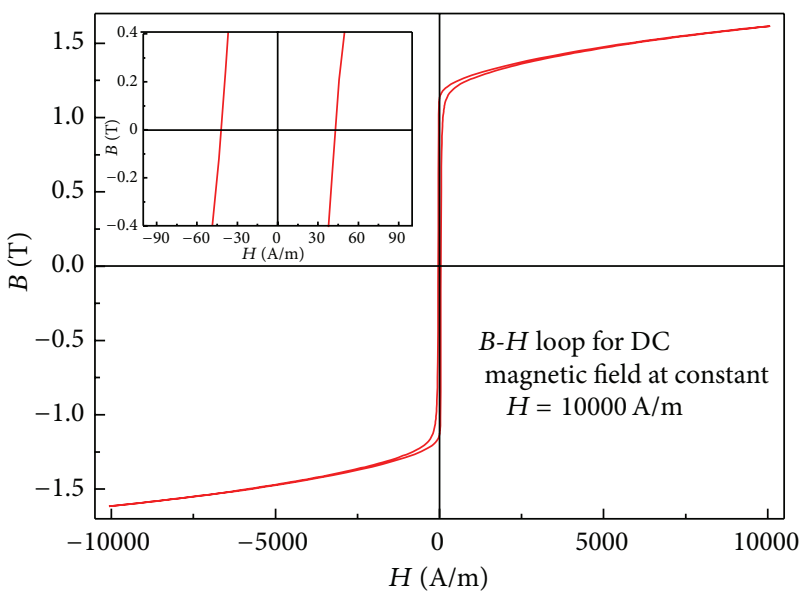

FIGURE 6: Static hysteresis loop of melt spinned ribbons after heat treatment $\left(1100^{\circ} \mathrm{C} / 5 \mathrm{~h}+\mathrm{FC}\right)$.

in the frequency range from $10 \mathrm{kHz}$ to $40 \mathrm{kHz}$, and the iron loss margin increases with enhancement of the frequency. In other words, the melt spinned ribbons have more advantage to be used in medium and high frequencies.

Figure 7 shows core loss curves of melt spinned ribbons. The iron loss is increased with frequency and magnetic induction intensity. The maximum permeability $\mu_{r}$ decreases with increase of frequencies as shown in Figure 8.

3.4. Core Loss Behavior. In general, the hysteresis loss dominates at low frequencies, while the eddy current loss and excess loss dominate at high frequencies. Separation of iron loss is important for magnetic materials to improve the quality and optimize the application conditions (the maximum frequency and the maximum magnetic flux density), so as to improve the process parameters.

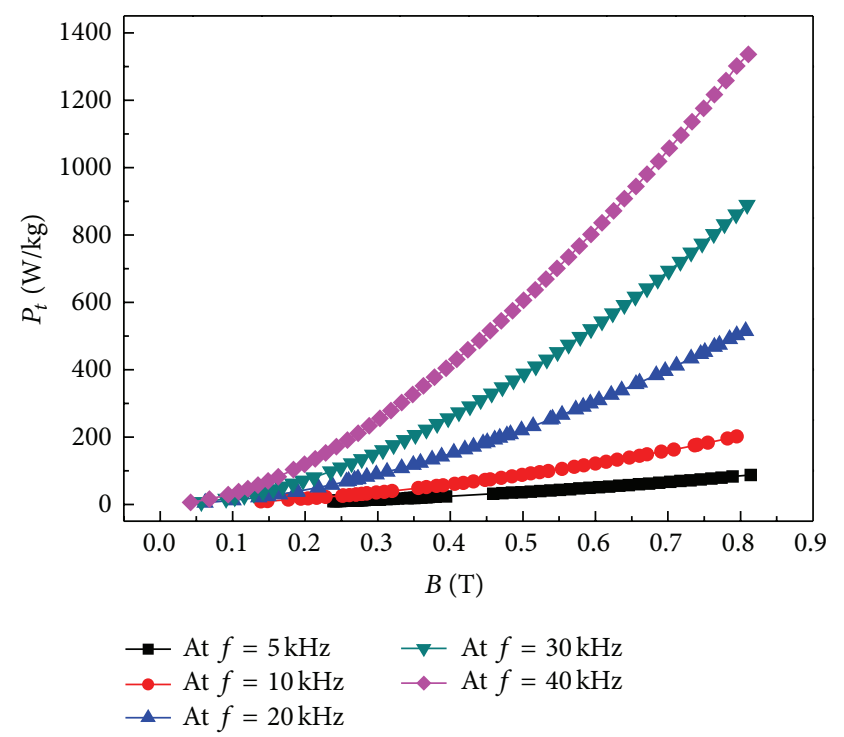

FIGURE 7: Core losses of melt spinned ribbons dependent on saturation magnetic induction $(B)$ and frequency $(f)$.

Iron loss can be represented by (1). Primarily, power losses can be separated into hysteresis loss $\left(P_{\text {hys }}\right)$, eddy current loss $\left(P_{e}\right)$, and excess loss $\left(P_{\text {exc }}\right)$ :

$$
P_{t}=P_{\mathrm{hys}}+P_{e}+P_{\mathrm{exc}} \cdot
$$

The hysteresis loss was quantified by Steinmetz [10] in his hysteresis loss model, which is given by

$$
P_{\text {hys }}=k_{h} B_{m}^{n} f \text {, }
$$

where $k_{h}$ is the hysteresis loss coefficient, $f$ is the frequency, and $n$ is the exponent of maximum magnetic induction intensity $B_{m}$, and its value is generally from 1 to 2 . 


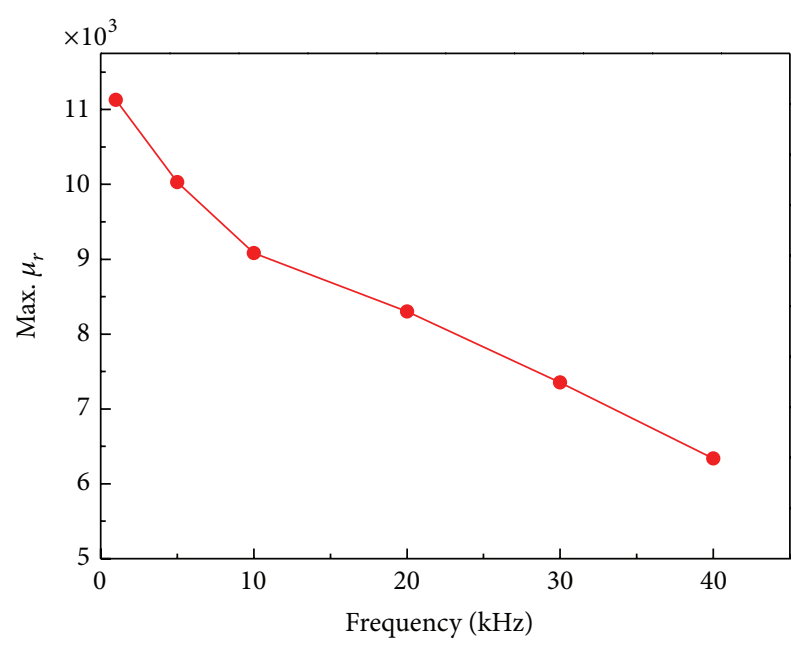

FIgURE 8: Variation of maximum permeability $\mu_{r}$ with frequency.

The eddy current loss $P_{e}$ can be explained by simple "classical" model [11, 12] using Maxwell's electrodynamics, which is given by

$$
P_{e}=\frac{\pi^{2} d^{2} B_{m}^{2} f^{2}}{\beta \rho},
$$

where $d$ is the thickness of the magnetic material ribbon, $f$ is the frequency, $\rho$ is the resistivity, and $\beta$ is the geometric factor.

There is great progress in measurement of excess loss using the statistical loss models [13-15], where magnetization dynamics were explained by statistics of a group of interacting domain walls, termed as magnetic objects (MO). Excess loss $\left(P_{\text {exc }}\right)$ can be given by

$$
P_{\text {exc }}=k_{\text {exc }} B^{3 / 2} f^{3 / 2} .
$$

Using all these models, namely, Steinmetz hysteresis model, classical eddy current loss mode, and statistical excess loss model, the total core loss can be written as

$$
P_{t}=k_{h} B^{n} f+k_{e} B^{2} f^{2}+k_{\mathrm{exc}} B^{3 / 2} f^{3 / 2},
$$

where $k_{h}, k_{e}$, and $k_{\text {exc }}$ are, respectively, hysteresis, eddy current, and excess loss coefficient.

To identify the values of the coefficients, (5) is divided by the frequency resulting in

$$
P_{\mathrm{cy}}=\frac{P_{t}}{f}=k_{h} B^{n}+k_{e} B^{2} f+k_{\mathrm{exc}} B^{3 / 2} f^{1 / 2},
$$

where $P_{c y}$ is the power loss per cycle. Equation (6) shows that hysteresis power loss per cycle does not depend on frequency. In this paper, the loss coefficients $\left(k_{h}, k_{e}\right.$, and $\left.k_{\text {exc }}\right)$ and exponent of $B$ (" $n$ ") have been determined by fitting of (6) within frequencies from $400 \mathrm{~Hz}$ to $40 \mathrm{kHz}$.

The $k_{h}$ and " $n$ " were determined by least square fitting, which is shown in Figure 9. The exponent " $n$ " value is 1.78 and $k_{h}$ value is 115.7. To further determine $k_{e}$ and $k_{\text {exc }}$, the " $n$ " and $k_{h}$ are put into (6). The modified (6) is written as

$$
P_{\mathrm{cy}}=115.7 B^{1.78}+k_{e} B^{2} f+k_{\mathrm{exc}} B^{3 / 2} f^{1 / 2} .
$$

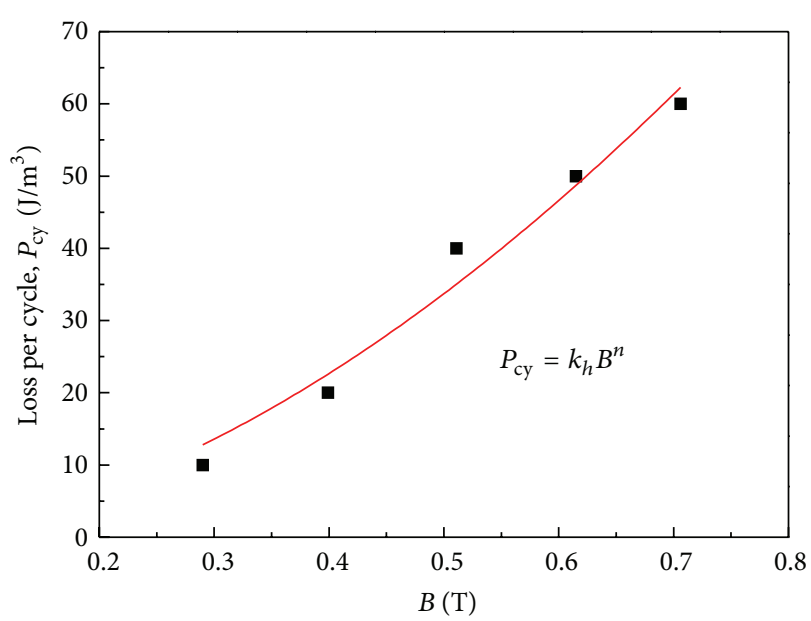

FIgURE 9: Variation of loss per cycle with $B$ at DC magnetic field.

Equation (7) is then fitted with $P_{\text {cy }}$-frequency curves at constant $B$. The obtained $k_{e}$ and $k_{\text {exc }}$ are plotted in Figure 10. It is found that the value of $k_{e}$ and $k_{\text {exc }}$ depend on the value of $B$, the value of $k_{e}$ is between 0.002 and 0.0043 , and the value of $k_{\text {exc }}$ increases with increasing $B$ value, leading to larger excess core losses at larger $B$ values, as shown in Figure 11.

The $k_{h}, k_{e}, k_{\text {exc }}$, and " $n$ " are put into (6) and then proportions of hysteresis loss $\left(P_{\text {hys }}\right)$, eddy current loss $\left(P_{e}\right)$, and excess loss $\left(P_{\text {exc }}\right)$ can be calculated at different conditions. Based on this result, people can adjust the production process and optimize heat treatment parameters to decrease the core loss.

\section{Conclusions}

(1) Fe-6.5wt.\%Si ribbons are prepared by melt spinning technique. Compared with cold rolled and CVD ribbons, the melt spinned ribbons possess much lower core losses at medium and high frequencies.

(2) Magnetic properties of the as-spinned ribbons are improved after heat treatment of $1100^{\circ} \mathrm{C} / 5 \mathrm{~h}$ with furnace cooling. Phase transformation of A2 to B2 is obvious after heat treatment.

(3) The loss coefficients are dependent on the flux density and the frequency. The value of $k_{e}$ is between 0.002 and 0.0043 , and $k_{\text {exc }}$ increases with $B$ value, leading to larger excess core losses at larger $B$ values.

\section{Conflict of Interests}

The authors declare that there is no conflict of interests regarding the publication of this paper.

\section{Acknowledgments}

Financial support from the Major State Basic Research Development Program of China (973 Program, no. 2011CB606304), the High-tech Research and Development Program of China 

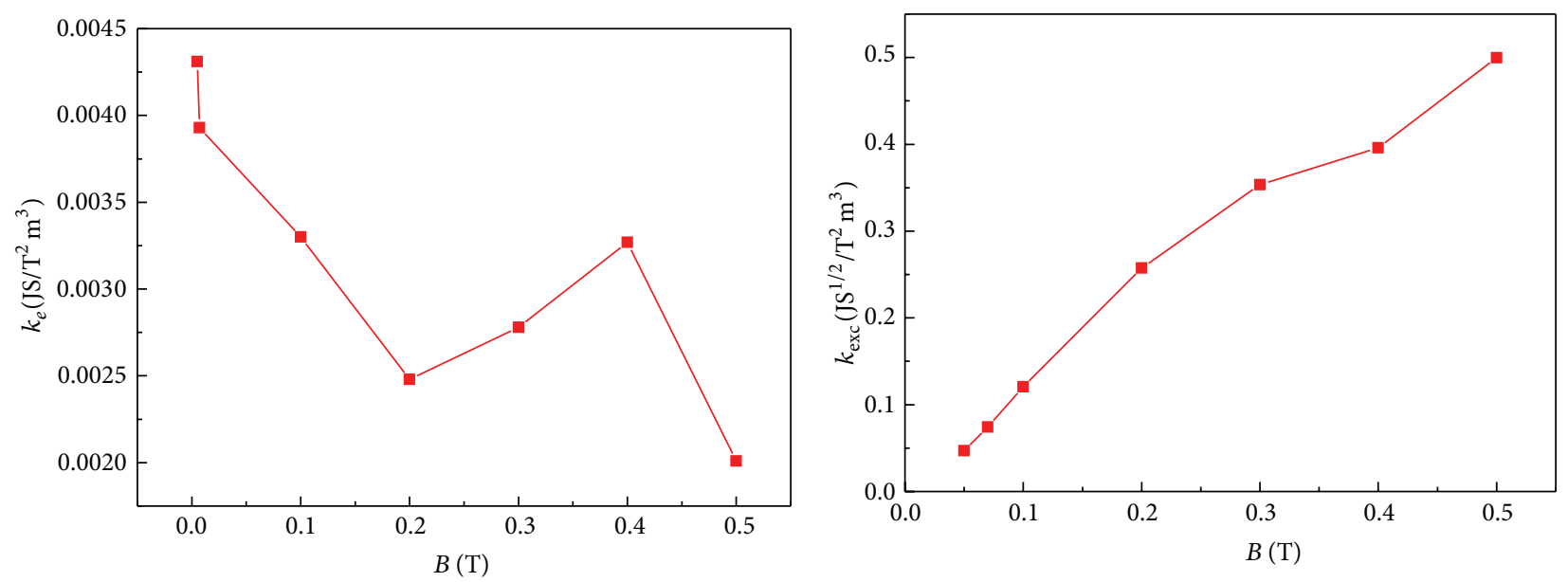

FIGURE 10: Variation of eddy current and excess loss coefficients $\left(k_{e}\right.$ and $\left.k_{\text {exc }}\right)$ with flux density $B$.

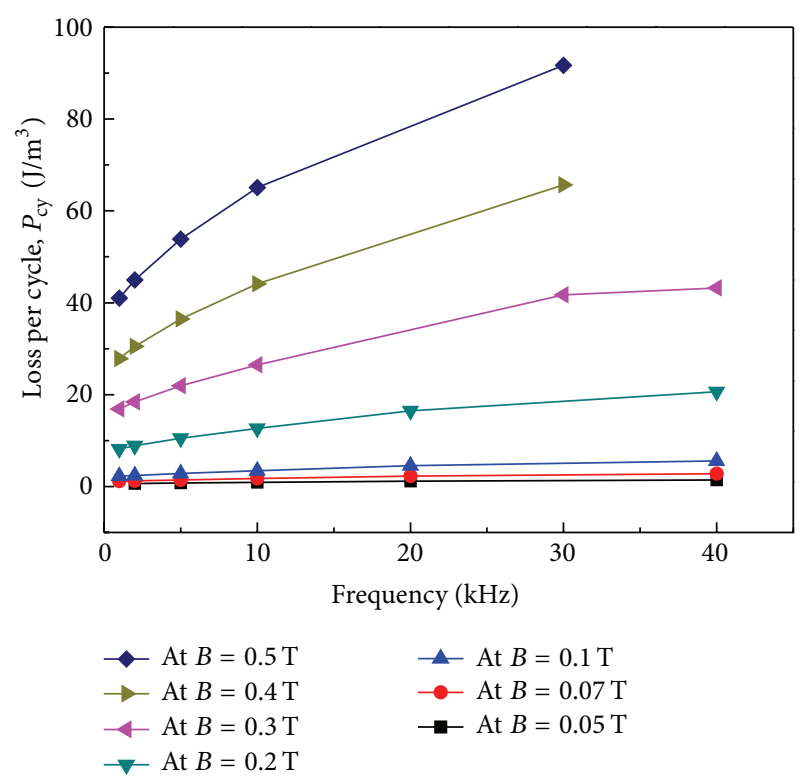

FIGURE 11: Variation of loss per cycle $\left(P_{\text {cy }}\right)$ with frequency at different $B$ values.

(863 Program, no. 2012AA03A505), the National Natural Science Foundation of China (no. 51301019), and Fundamental Research Funds for the Central Universities (no. FRF-TP-14099A2) is gratefully acknowledged.

\section{References}

[1] K. Narita, N. Teshima, Y. Mori, and M. Enokizono, "Recent researches on high silicon-iron alloys," IEEE Transactions on Magnetics, vol. 17, pp. 2857-2862, 1981.

[2] P. R. Swann, L. Grånäs, and B. Lehtinen, "The $\mathrm{B2}$ and $\mathrm{DO}_{3}$ ordering reactions in iron-silicon alloys in the vicinity of the curie temperature," Metal Science, vol. 9, no. 1, pp. 90-96, 1975.

[3] B. Viala, J. Degauque, M. Fagot, M. Baricco, E. Ferrara, and F. Fiorillo, "Study of the brittle behaviour of annealed Fe-6.5 wt $\% \mathrm{Si}$ ribbons produced by planar flow casting," Materials Science and Engineering A, vol. 212, no. 1, pp. 62-68, 1996.

[4] H. Haiji, K. Okada, T. Hiratani, A. Abe, and M. Ninomiya, "Magnetic properties and workability of $6.5 \%$ Si steel sheet," Journal of Magnetism and Magnetic Materials, vol. 160, pp. 109114, 1996.

[5] M. Enokizono, N. Teshima, and K. Narita, "Magnetic properties of 6.5 percent silicon-iron ribbon formed by a melt spinning technique," IEEE Transactions on Magnetics, vol. 18, no. 5, pp. 1007-1013, 1982.

[6] N. Tsuya, K. Arai, and K. Ohmori, "Ribbon-form sendust alloy," IEEE Transactions on Magnetics, vol. 15, no. 4, pp. 1149-1153, 1979.

[7] K. Raviprasad, M. Tenwick, H. A. Davies, and K. Chattopadhyay, "The nature of ordered structures in melt spun iron-silicon alloys," Scripta Metallurgica, vol. 20, no. 9, pp. 1265-1270, 1986.

[8] K. Narita and M. Enokizono, "Effect of ordering on magnetic properties of 6.5-percent silicon-iron alloy," IEEE Transactions on Magnetics, vol. 15, no. 1, pp. 911-915, 1979.

[9] Y. F. Liang, F. Ye, J. P. Lin, Y. L. Wang, and G. L. Chen, "Effect of annealing temperature on magnetic properties of cold rolled high silicon steel thin sheet," Journal of Alloys and Compounds, vol. 491, no. 1, pp. 268-270, 2010.

[10] J. E. Brittain, "A steinmetz contribution to the AC power revolution," Proceedings of the IEEE, vol. 72, no. 2, pp. 196-221, 1984.

[11] C. D. Gmham, "Physical origin of losses in conducting ferromagnetic materials (invited)," Journal of Applied Physics, vol. 53, pp. 8276-8280, 1982.

[12] E. W. Golding, Electrical Measurements and Measuring Instruments, Pitman and Sons Limited, London, UK, 4th edition, 1961.

[13] G. Bertotti, "General properties of power losses in soft ferromagnetic materials," IEEE Transactions on Magnetics, vol. 24, no. 1, pp. 621-630, 1987.

[14] G. Bertotti, "A general statistical approach to the problem of eddy current losses," Journal of Magnetism and Magnetic Materials, vol. 41, pp. 253-260, 1984.

[15] O. de la Barrière, C. Appino, F. Fiorillo et al., "Loss separation in soft magnetic composites," Journal of Applied Physics, vol. 109, no. 7, Article ID 07A317, 2011. 

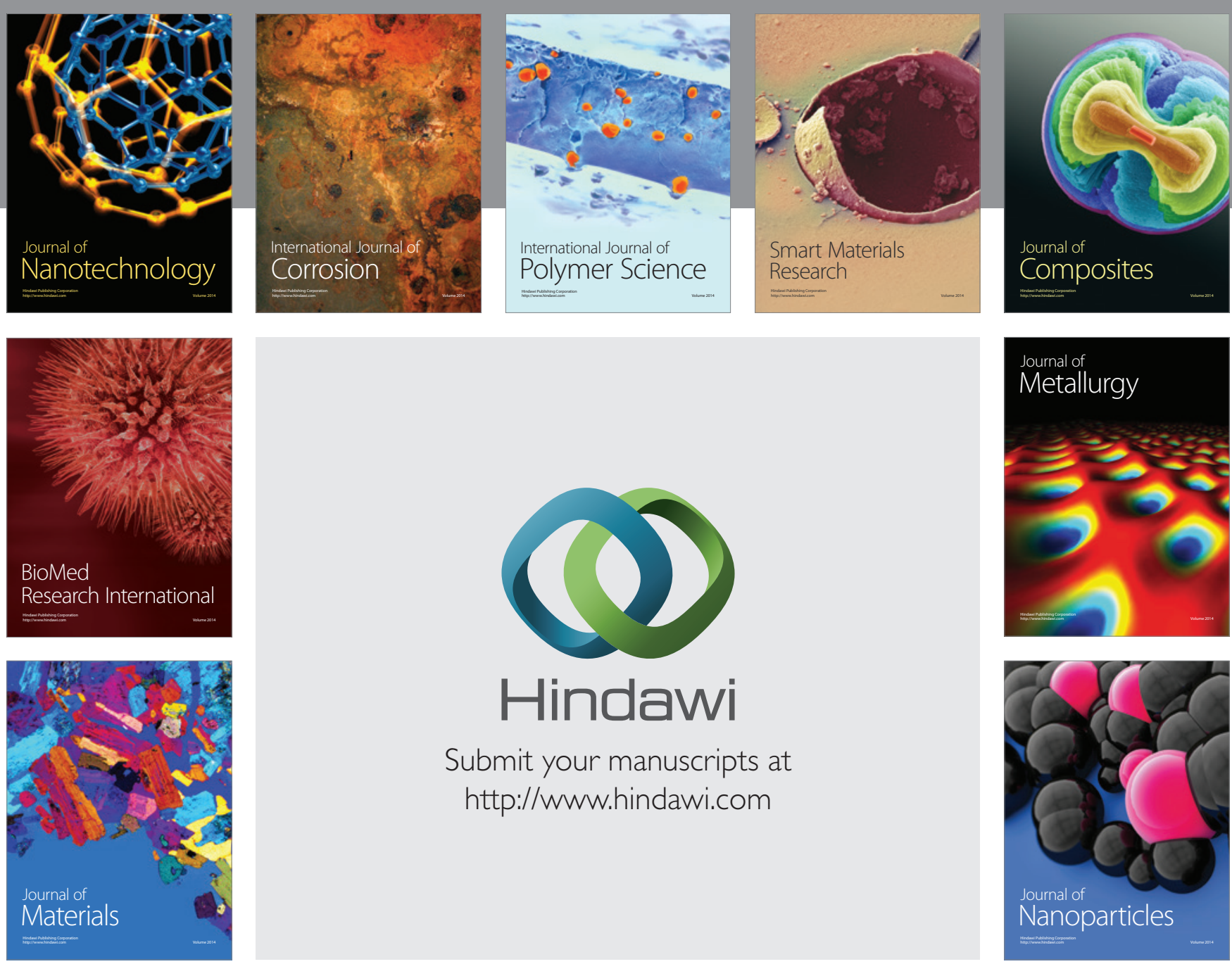

Submit your manuscripts at http://www.hindawi.com
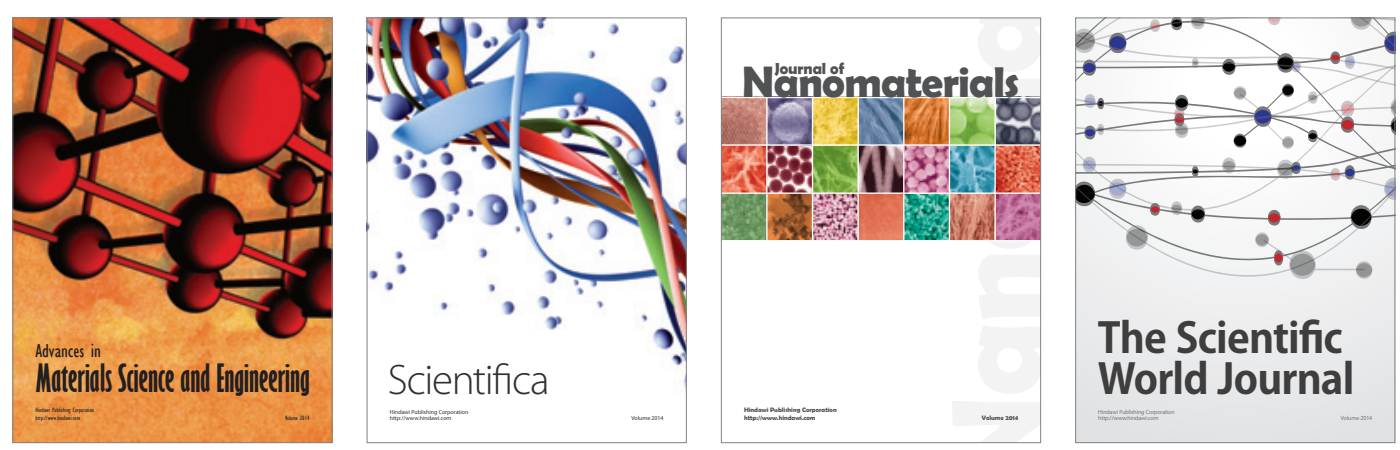

\section{The Scientific World Journal}
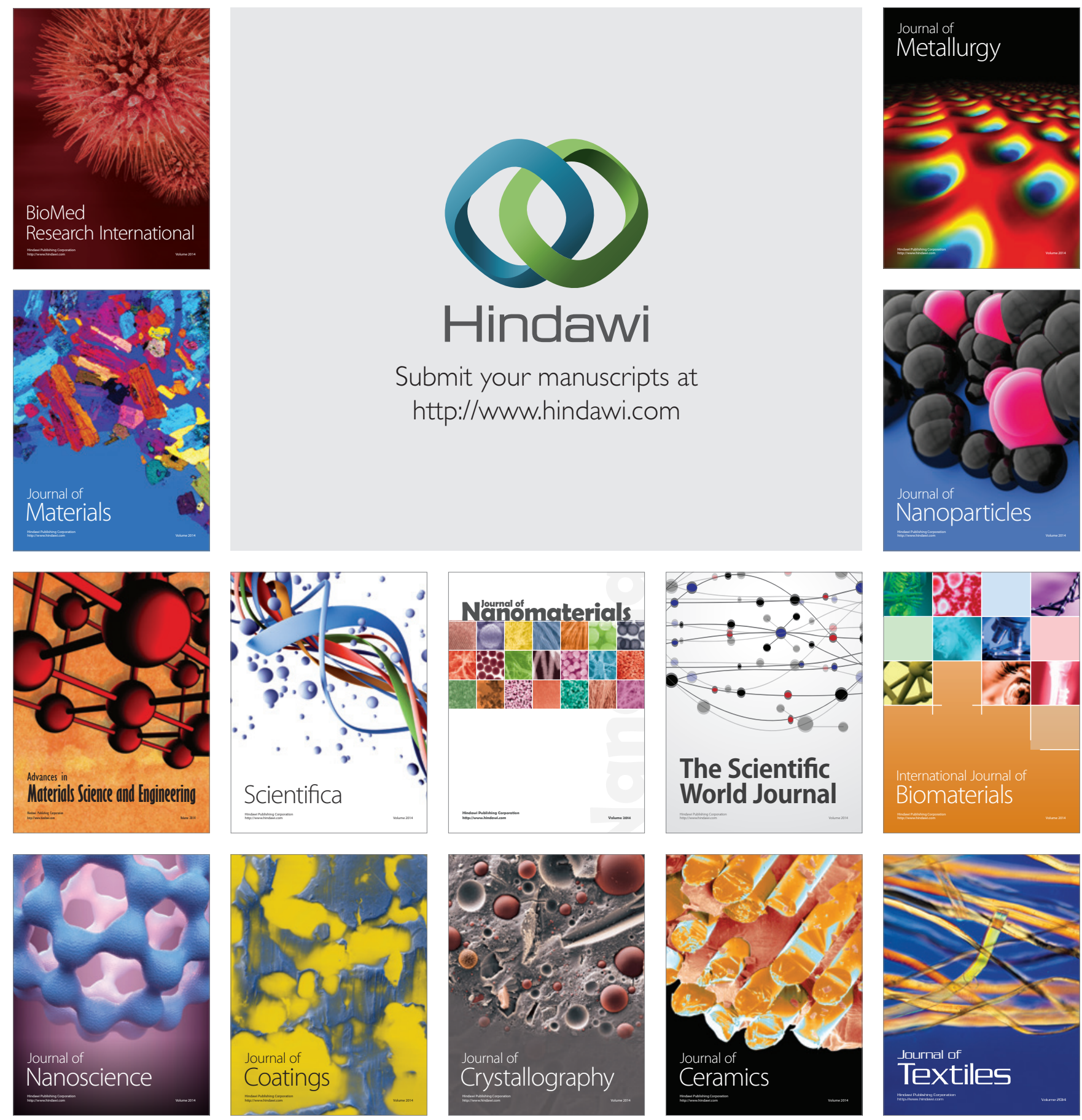